\title{
Tiempos normales o posnormales para la Ciencia de la Información
}

\section{Nancy Blanco}

Universidad de Buenos Aires. Facultad de Filosofia y Letras. Departamento de Bibliotecologìa y Ciencia de la Información. Buenos Aires, Argentina. Universidad de Buenos Aires. Facultad de Filosofía y Letras. Instituto de Investigaciones Bibliotecológicas (INIBI). Buenos Aires, Argentina / nancybl@filo.uba.ar / https://orcid.org/oooo-0oo2-4218-6187

\begin{abstract}
Resumen
En este Editorial se realiza una revisión de las perspectivas que intentan describir los conceptos producidos en la investigación científica y tecnológica en relación con la "ciencia posnormal". Estos estudios han tenido un gran impacto en los últimos años y su abordaje en sus aportes más destacables presuponen repensar a la disciplina como productora de conocimiento. Se asocian estas transformaciones a un mayor acercamiento de la ciencia hacia los intereses y preferencias de sus principales actores sociales. La ciencia de la información, interpelada por la ciencia posnormal, en el sentido que a este último término le atribuyeran Silvio Funtowicz y Jerome Ravetz, se enfrenta a situaciones en las que el encuentro con la información, el conocimiento y la sabiduría se brindan en contextos complejos, donde los marcos conceptuales que estructuran la perspectiva del bibliotecario deben coevolucionar para no permanecer inalterables.
\end{abstract}

\section{Normal or post-normal times for Information Science}

\begin{abstract}
This Editorial is a review of the perspectives that attempt to describe the concepts produced in scientific and technological research in relation to "post-normal science". These studies have had a great impact in recent years and their approach, in their most notable contributions, presuppose rethinking the discipline as a producer of knowledge. These transformations are associated with a closer approach of science towards the interests and preferences of its main social actors. Information science, challenged by post-normal science, in the sense that Silvio Funtowicz and Jerome Ravetz attributed to the latter term, faces situations in which the encounter with information, knowledge and wisdom are provided in complex contexts, where the conceptual frameworks that structure the librarian's perspective must coevolve in order not to remain unchanged.
\end{abstract}

\section{Keywords}

Post-normal science Library science Information science Wisdom Knowledge 
En esta dimensión la Facultad de Ciencias Sociales, la Facultad de Filosofía y Letras de la Universidad de Buenos Aires, el Centro Redes y el Ministerio de Ciencia y Tecnología de la provincia de Córdoba organizaron un coloquio en diciembre del 2020 titulado Ciencia posnormal en acción cuyo principal disertante fue Silvio Funtowicz. Durante la apertura, se señaló que la ciencia posnormal propone abordar problemas complejos de producción, uso científico y tecnologías que requieren decisiones acertadas bajo contextos de alta incertidumbre y riesgos.

El control de la calidad del conocimiento científico presupone que se dirija al proceso de toma de decisiones y que se involucre no solo a la información científica, sino a la sociedad en general, dado que este tipo de problemas afecta global y localmente a la ciudadanía como lo fue la pandemia en este último año. Por este motivo, resulta importante la vinculación de valores, decisiones y experticia entre gobiernos, comunidades disciplinarias, analistas de la ciencia y la ciudadanía en general (Funtowicz, 2020).

Durante la conferencia Funtowicz explicó los alcances que propone este enfoque. La ciencia posnormal señala que los desafíos son complejos y ambiguos, y en la coexistencia de una pluralidad de perspectivas que son legítimas debe evitarse el reducir unas a las otras. La ciencia posnormal no implica un nuevo paradigma científico, tampoco se presenta como un nuevo método, ni aspira a convertirse en una nueva normalidad, se propone como una visión acerca del rol del conocimiento de la ciencia en la sociedad.

Una cuestión se define como posnormal cuando los hechos se presentan como inciertos, la pluralidad de valores se encuentran en conflicto, la puesta en juego resulta potencialmente elevada y las decisiones son urgentes (Funtowicz, 2020). Este concepto considera que los problemas de la realidad contemporánea involucran a sistemas complejos que pueden carecer de una respuesta científica o tener más de una. En este escenario, la verdad se transfiere a la calidad cuando se unen los hechos con los valores y se toman decisiones en la acción social.

Otros autores como Jiménez-Buedo y Ramos Vielba (2009) aclaran que en la propuesta de ciencia posnormal de Funtowitz se reconoce a la incertidumbre como inherente de los sistemas complejos por lo que, en este punto, la toma de decisiones resulta inevitable. La ciencia posnormal no cuestiona los fundamentos de la ciencia tradicional sino más bien conlleva una nueva forma organizativa.

La visión de la ciencia depende de varios factores, algunos internos que hacen a sus organizaciones como el quehacer de los científicos, y otros externos que resultan ser determinantes de su carácter social. En esta doble perspectiva se vincula las interacciones entre ciencia y sociedad. Sin embargo, al pensar a la ciencia como un sistema estático puede carecer de herramientas que den respuesta a la complejidad de las vinculaciones multidireccionales y las dinámicas que la relacionan con la ciencia básica, aplicada y el desarrollo tecnológico (Jiménez-Buedo y Ramos Vielba, 2009).

En el campo de la comunicación Brüggemann, Lörcher y Walter (2020) sugieren que la aplicación del concepto de ciencia posnormal de Funtowicz y Ravetz puede ayudar a analizar los cambios emergentes. En este sentido, explican que la ciencia se encuentra desafiada por situaciones posnormales. La primera, se origina cuando la ciencia produce altos niveles de incertidumbre y un grado considerable de desconocimiento debido a la falta de datos, la complejidad del tema, o un enfoque en proyecciones de riesgos futuros. Un segundo escenario se muestra cuando al desarrollar políticas, con base científica, se involucran cuestiones de alto valor que no pueden responderse mediante la investigación. La tercera circunstancia plantea que los temas en cuestión son relevantes para la sociedad e implican altos costos y finalmente se presenta, 
cuando la toma de decisiones políticas resulta urgente (Funtowicz y Ravetz, 1994). En estas circunstancias, la ciencia posnormal, como marco, colabora en comprender cómo reacciona la ciencia ante estas situaciones.

Post, Bienzeisler y Lohöfener (2021) sostienen que la pandemia del coronavirus creó una situación que los investigadores han denominado posnormal, en contraste con el concepto clásico de ciencia normal. Esta última, denota un esfuerzo a largo plazo en el que los científicos se comprometen a ampliar y perfeccionar el conocimiento existente, como independiente de los problemas de la sociedad. En este proceso, los científicos suelen identificar y resolver cuestiones de investigación aisladas y cada vez más específicas, mientras que otras preocupaciones que la sociedad considera urgentes, una cura para el cáncer o el diseño de una paz duradera, a menudo quedan en enigmas, en gran parte porque pueden carecer de una pronta solución (Kuhn, 2004). La ciencia posnormal se encuentra en un período de transición de su modelo que puede resultar incomprendido por los científicos formados en el paradigma anterior y dominante.

La ciencia posnormal surge, entonces, en crisis sociales urgentes, cuando el conocimiento científico disponible resulta incierto e incompleto. La pandemia del coronavirus mostró una situación en la que el conocimiento virológico y epidemiológico resultó escaso, y los conflictos de valores debían resolverse, entre otros, en lo que respecta a las libertades personales o a la protección de los vulnerables. En estas circunstancias, la erudición científica estuvo fuertemente orientada hacia las necesidades de la gestión de la crisis, sin embargo, dejó el interrogante sobre cómo trasladar estas cuestiones en la toma de decisiones (Post, Bienzeisler y Lohöfener, 2021; Brüggemann, Lörcher y Walter, 2020; Funtowicz y Ravetz, 1994).

En la ciencia y la tecnología (ciencia aplicada), la investigación tradicional se presentacomo jerárquica, homogénea y diciplinar, su control de calidad recae en la comunidad de pares y los resultados obtenidos quedan en dominio público. La investigación actual, se caracteriza por ser transdisciplinar, heterogénea, heterárquica, y además priman dos factores que buscan satisfacer demandas sociales específicas: su aplicabilidad y su utilidad social. En este sentido, se busca que la producción del conocimiento se encuentre orientada al contexto de aplicación y la validación de los resultados se apoye en la aceptación social (Jiménez-Buedo y Ramos Vielba, 2009).

La comunicación según Méndez (2021) en estos escenarios, se produce desde la sociedad hacia los productores de conocimiento. Las soluciones a los problemas de interés social se mantienen en una permeabilidad constante entre las demandas y las necesidades, y la valoración de la ciencia se funda en los conocimientos útiles para la vida de los ciudadanos. El criterio rector, en estos casos, se enmarca como utilitario y se actúa en un marco de conocimiento distribuido socialmente (Jiménez-Buedo y Ramos Vielba, 2009).

En esta coevolución entre ciencia y sociedad Taddei e Hidalgo (2016) sostienen que las decisiones deberían ser tomadas por una comunidad de pares extendida, que incluya a todos los afectados por el problema, de esta manera se reconoce la pluralidad de perspectivas legítimas acerca de una cuestión. La realidad social y naturalmente cambiante hace comprender que el contexto en el cual se diseñó ha sido desorganizado, "entender que no es necesariamente el mundo el que está 'dislocado' sino que el contexto en el que se diseñó su 'transcripción' científica ha sido desorganizado por una realidad social y natural cambiante" (Taddei e Hidalgo, 2016: 28)

Pérez Flores (2015) destaca que las tecnologías de la información juegan un papel fundamental en las sociedades altamente globalizadas. De acuerdo con Functowicz y 
Ravetz la ciencia posnormal en las nuevas formas de producción y democratización del conocimiento se presentan reflexivamente en los niveles técnico, metodológico y epistemológico. Pérez Flores, cita al filósofo León Olivé quien señala a la ciencia como una práctica social y destaca que existen tres imágenes que muestran su pluralismo. La primera se vincula a los grupos de científicos, la segunda se muestra como la representación externa de las distintas disciplinas sobre la actividad científica y, finalmente se destaca la imagen pública de la ciencia, que refiere a la percepción generalizada en la sociedad. Esta última carece de ciudadanos pasivos ya que se compone de múltiples actores reflexivos.

La ciencia ciudadana encuentra otro espacio en el artículo de Méndez en el cual se sostiene que un componente normativo de fondo de la Open Science formula "que los ciudadanos puedan contribuir de manera significativa en la investigación y que sean reconocidos como productores de conocimiento en la ciencia europea" (Méndez, 2021: 9). Además, agrega que el cambio cultural necesario se producirá en la confluencia de aproximaciones políticas (top-down) sin dejar de lado los compromisos prácticos de implementación (bottom-up). De esta forma propone que la ciencia sea capaz, no sólo de responder preguntas y crear informes, sino de resolver problemas.

Teóricos en conocimiento, ciencia y democracia han argumentado que, en la formulación de políticas basadas en ciencia, la distinción entre estas debe ser clara para permitir que los ciudadanos reconozcan los hechos científicos y se involucren en los debates sobre valores y prioridades sociales. Desde el punto de vista empírico, sin embargo, se analizó que se tienen fuertes preferencias por delegar la toma de decisiones a científicos o círculos de expertos en aras de la eficiencia. Señalan, además, que se ha observado una visión ingenua, es decir, lineal del vínculo entre el conocimiento científico y la toma de decisiones. Esta última, necesariamente implica juicios de valor, por ejemplo, al priorizar los resultados deseados o al calcular los costos y beneficios de ciertas medidas. En este aspecto, la función de la ciencia en los debates debería ir más allá de prescribir, dado que conlleva el compromiso de informar sobre la toma de decisiones e identificar y comparar opciones específicas entre sí de manera sistemática (Post, Bienzeisler y Lohöfener, 2021).

En otras reflexiones se menciona que las personas tienen diferentes conceptos sobre la naturaleza del conocimiento científico, es decir, creencias epistémicas. Algunos sostienen dogmas epistémicos más sofisticados que distinguen el conocimiento científico como provisional y las teorías revisables, mientras que otros poseen ideales que perciben a estas últimas como invariables y al conocimiento como incuestionable. Una visión del saber científico como cierto y estable corresponde a una visión lineal de la relación entre ciencia y política que los pensadores más teóricos han descartado como simplista. Las investigaciones han encontrado que cuanto más se suscribe a la creencia de que el conocimiento científico es definitivo, más se delega a la autoridad científica la toma de decisiones sociales en ciencia y tecnología (Howell et al., 2020).

La sociedad en general aprecia la participación de los científicos en la toma de decisiones, ya que están a favor de delegarlas en círculos de expertos. Estas se basan, en algunos casos, en que se prefiere la eficiencia y en otros, en que se legitima que los eruditos pueden decidir mejor lo que es bueno para la población. Otras teorías sobre conocimiento y democracia plantean que los actores en ciencia deben promover sus dominios de investigación para que los ciudadanos puedan involucrarse en los debates sobre valores y prioridades. El grado en que esto resulte factible depende, entre otras cuestiones, de las conceptualizaciones ciudadanas sobre la relación entre ciencia, política y debate público (Post, Bienzeisler y Lohöfener, 2021). 
Para los ciudadanos, la pandemia del coronavirus creó una gran crisis social que podría afectar la salud personal y el bienestar psicológico y económico con un resultado muy incierto. En estas situaciones, las personas tienen una gran necesidad de información. Chew (1994) presenta una diferencia entre una necesidad informativa orientativa y una constructiva.

Una necesidad informativa de orientación representa un deseo de información definido que potencialmente reduzca el vacío de conocimiento. Se relaciona con preguntas como ¿cuáles son los detalles de la situación? o ¿qué dicen los expertos? Por el contrario, una necesidad de construcción representa el deseo de diferentes puntos de vista para tomar una decisión. Se relaciona con preguntas como ¿cuáles son los diferentes puntos de vista para que pueda seleccionar la mejor posición? o ¿qué información me ayuda a desarrollar una opinión? (Chew, 1994).

Las opiniones de los ciudadanos en la toma de decisiones democráticas sugieren que las necesidades de información de los individuos se relacionan con sus puntos de vista normativos sobre la cooperación entre científicos en tiempos de crisis. La preferencia entre los ciudadanos es excluir el debate público y delegar la toma de decisiones políticas en círculos de expertos en aras de la eficiencia. En vista de estos hallazgos, se asume que las preferencias informativas de las personas en la pandemia están asociadas con sus expectativas normativas de la relación entre los científicos, con quienes buscan información definitiva para favorecer su orientación (Chew, 1994).

En este sentido para los autores, la pandemia planteó una situación muy incierta para la sociedad en general y para la vida cotidiana de las personas. Los individuos no solo tienen diferentes capacidades para tolerar la incertidumbre o las ambigüedades sino también distintas formas de afrontarlas. Mientras que algunos prefieren un conocimiento seguro y estable que no sea cuestionado otros escogen tomar decisiones basadas en información contrapuesta.

Uribe-Tirado et al. en su artículo Datos, información y conocimiento: otra cruel pedagogía del virus sostiene que se presenta una dependencia hacia los datos, pero en realidad lo que se necesita es información. La cantidad de datos durante la pandemia del Covid 19 simbolizó para la ciudadanía global una idea del estado de la situación en diferentes territorios. Sin embargo, el aprender solo de los datos y no de las relaciones entre estos con otros como por ejemplo las conceptualizaciones desde diferentes disciplinas, deja sin comprender el problema y en consecuencia genera prácticas de gestión complejas e ineficaces (Uribe-Tirado et al., 2020).

Datos, información, conocimiento, entendimiento y sabiduría (DICES) componen una herramienta para que los debates en ciencia puedan realizarse desde referentes conceptuales consistentes, coherentes y útiles. En este contexto un problema que se relacione con el procesamiento de datos debe apoyar el hallazgo de relaciones, patrones, principios o leyes. Las respuestas se constituyen en componentes para comprender la estructura y la dinámica que afecta a una realidad o un problema. La toma de decisiones en entornos de datos abundantes afecta a las instituciones y el tipo de acción que deben emprender. Por lo tanto, aquellas que resultan oportunas, acertadas, dirigidas a enfrentar fenómenos mundiales, conllevan a repensar que los datos y la información pueden convertirse en componentes esenciales de la solución al problema. Al acercar la sociedad al conocimiento, se intenta revisar en qué manera se desarrolla, comparte y aplica el conocimiento científico y tecnológico (Uribe-Tirado et al., 2020).

Navegar en tiempos posnormales según Sardar (2020) requiere un nuevo orden de sabiduría. Esta se podría definir simplemente como una toma de decisiones experta basada en la experiencia, la comprensión y el conocimiento. Sin embargo, la 
profundidad del conocimiento y la perspicacia para circunnavegar en aquel en que se basa en la falsificación de noticias, en los hechos alternativos, en la ciencia aparentemente fabricada, en las historias quiméricas anónimas en línea, resulta excesivo y complejo.

En este sentido, se podría repensar la sabiduría no como algo inherente al sujeto sino como una virtud asociada a la comunidad. Se presenta la necesidad de pasar de la noción convencional de sabiduría como depósito de la calidad individual, o como una prerrogativa de los individuos sabios, a una comprensión más profunda y holística: la sabiduría como una empresa colectiva y comunitaria (Sardar, 2020).

Sardar (2020) plantea que en tiempos posnormales, la sabiduría debe verse como una moral colectiva, un aprendizaje cooperativo racional sobre cómo vivir de manera sostenible, un esfuerzo comunitario para crear lo que es verdaderamente significativo; aquello que mejora la calidad de vida, el bienestar humano y, por último, lo que se proyecta como un futuro genuino de justicia e igualdad. El conocimiento emergente en este contexto será una combinación de conocimiento objetivo y aquel conocimiento tóxico. En relación con este último, Bawden y Robinson (2018) reconocen que la práctica de la ciencia de la información no solo resulta crucial para dirigir la preservación del registro humano, sino también debería ayudar en la búsqueda de su cometido para superar algunos de los problemas percibidos del entorno de la información actual entre estas, las noticias falsas, posverdad y hechos alternativos.

Maxwell (2007) proporciona una definición más amplia de sabiduría y tal vez más adecuada para esta época: la sabiduría incluye el conocimiento y la comprensión, pero va más allá al implicar además el deseo y la lucha activa por los valores. En este punto hace referencia a las capacidades en torno al valor: de observarlo, real y potencialmente en las circunstancias de la vida; de experimentarlo y de darse cuenta de lo que es importante para uno mismo y para los demás; de resolver o ayudar en los problemas de la vida que surgen al realizar aquello que es de valor; y finalmente de utilizar y desarrollar el conocimiento, la tecnología y la comprensión necesarios para la realización de lo que es de valor. Además, sugiere que tal sabiduría puede ser institucional y social y Sardar (2020) agrega que también debe ser comunitaria y estar en red. Así como el conocimiento que se adquiere en las comunidades de investigadores y académicos la sabiduría también tiene que ser generada por comunidades que compartan normas, valores y objetivos comunes.

La sabiduría comunitaria propuesta deberá contar con conocimiento genuino, es decir, conocimiento tácito o lo que se podría denominar también como conocimiento de mano. Este último, se encuentra incrustado culturalmente, tiene sentido y proporciona dirección dentro de un medio cultural particular. Se puede pensar como aquel de los pueblos originarios, las sociedades tradicionales y las comunidades académicas, donde se transmite de generación en generación. Se encuentra inerte en los individuos, que pueden no ser conscientes de que lo poseen, y hallarse compartido en las comunidades. Al igual que la capacidad de hablar urdu, tocar el sitar o diseñar edificios y ciudades, se define como complejo, abstracto, arraigado, profundamente causal, difícil de articular y, como tal, resulta complicada su trasferencia a otras personas, excepto cuando estas se unen a la comunidad (Sardar, 2020; Taddei e Hidalgo, 2016).

El conocimiento tácito goza de una naturaleza más íntima que se adquiere mediante la escucha profunda, la reflexión interior, la especulación metafísica, la comunión con la naturaleza, es decir, se exterioriza como una percepción y un entendimiento etéreo que se puede poseer en la palma de la mano. Se relaciona con el tipo de conocimiento que une a las personas para la erudición mutua, el cuidado, la curación 
y el crecimiento. Permite que se generen espacios entre los individuos, para que estos aprendan unos de otros y que compartan sabiduría tan antigua, pero a la vez contemporánea, un tipo de sabiduría que es capaz de crear redes entre las personas, la tierra, y las culturas (Sardar, 2020).

Además de las virtudes establecidas, las comunidades de sabiduría también tendrán una virtud compleja y holística esencial para los tiempos posnormales o lo que Vallor llama virtud tecnomoral, entendida como la capacidad de ver en la dimensión moral el acelerado cambio tecnológico. La virtud tecnomoral se interpreta como algo diferente de las virtudes establecidas como la honestidad, la flexibilidad, la humildad y el autocontrol ya que no se define como un temperamento preciso, sino como una condición general de experticia moral cultivada e integrada. Funciona como una lente a través de la cual se contextualiza y cultivan viejas virtudes con una adaptación nueva y explícita al emergente entorno tecnológico y global. La virtud tecnomoral funciona entonces, como un intelecto colectivo que permite observar lo positivo en un contexto cambiante y elegir futuros viables (Vallor, 2018).

El análisis de la ciencia desde la perspectiva global y en su evolución, donde se sitúa no solo al sujeto sino también en su contexto en sociedad, propone en su revisión mirar a la disciplina más allá desde una secuencia lineal escalonada, y repensarla hacia un movimiento de apertura donde coexisten en tensión las distintas propuestas, sin absolutizar ni eliminar los constructos ya establecidos. En este enfoque, los consensos trascienden el ámbito disciplinar y buscan la flexibilidad para intentar dar respuesta a los problemas científicos y sociales.

La ciencia de la información se presenta como una posibilidad en la reconstrucción del vínculo entre la ciencia y la sociedad, desde su papel en la formación crítica de los usuarios, en la educación de profesionales reflexivos ante la posverdad y en la alfabetización digital para comprender la necesidad de una ciencia posnormal. El papel del profesional de la información y su compromiso con la ciudadanía, al acercar la información y el conocimiento a la sociedad, le permitirá encontrar no solo las respuestas a las preguntas de los problemas que se presentan en este período posnormal sino, además, le concederá la posibilidad de alimentar esa sabiduría colectiva tan necesaria. En palabras de Funtowicz la ciencia no habla con una sola voz, las comunidades extendidas de pares que participan plenamente en la definición y resolución de los problemas y en la evaluación de la calidad de las propuestas cumplen la función de un propósito 'socialmente construido'. 


\section{Q Referencias bibliográficas}

"Bawden, David y Lyn Robinson. 2018. Curating the infosphere: Luciano Floridi's Philosophy of Information as the foundation for Library and Information Science. En Journal of Documentation. Vol. 74, no. 1, 2-17. <https://doi.org/10.1108/ JD-07-2017-0096>

» Brüggemann, Michael, Ines Lörcher y Stefanie Walter. 2020. Post-normal science communication: exploring the blurring boundaries of science and journalism. En Journal of Science Communication. Vol. 19, no. 3, Ao2. <https://doi. org/10.22323/2.19030202>

"Chew, Fiona. 1994. The Relationship of Information Needs to Issue Relevance and Media Use. En Journalism Quarterly. Vol. 71, no. 3, 676-688. <https://doi. org/10.1177/107769909407100318>

» Funtowicz, Silvio. 2020. Ciencia Posnormal en acción. Ministerio de Ciencia y Tecnología. <https://youtu.be/l_1nW_wwP2Y> [Consulta: 20 octubre 2021].

» Funtowicz, Silvio O. y Jerome R. Ravetz. 1994. Uncertainty, complexity and post normal science. En Environmental Toxicology and Chemistry: An International Journal. Vol. 13, no. 12, 1881-1885. <https://doi.org/10.1002/etc.5620131203>

" Funtowicz, Silvio O. y Jerome R. Ravetz. 2019. Science for the post-normal age. En Sardar, Ziauddin, ed. The Postnormal Times Reader. Washington DC: International Institute of Islamic Thought. p. 23-46. <http://www.jstor.org/stable/j. ctv10kmcqv.4> [Consulta: 20 octubre 2021].

» Howell, Emily L., Christopher D. Wirz, Dietram A. Scheufele, Dominique Brossard y Michael A. Xenos. 2020. Deference and decision-making in science and society: How deference to scientific authority goes beyond confidence in science and scientists to become authoritarianism. En Public Understanding of Science. Vol. 29, no. 8, 80o-818. <https://doi.org/10.1177/0963662520962741>

" Jiménez-Buedo, María e Irene Ramos Vielba. 2009. ¿Más allá de la ciencia académica?: Modo 2, ciencia posnormal y ciencia posacadémica. En ARBOR Ciencia, Pensamiento y Cultura. Vol. 185, no. 738, 721-737. <https://doi.org/10.3989/ arbor.2009.738n1048>

» Kuhn, Thomas S. 2004. La estructura de las revoluciones científicas. México: Fondo de Cultura Económica.

"Maxwell, Nicholas. 2007. From Knowledge to Wisdom : A Revolution for Science and the Humanities. London: Pentire Press.

"Méndez, Eva. 2021. Open Science por defecto. La nueva normalidad para la investigación. En ARBOR Ciencia, Pensamiento y Cultura. Vol. 197, no. 799, a587. <https://doi.org/10.3989/arbor.2021.799002>

»Pérez Flores, Rosa Elena. 2015. Modernidad, reflexividad y ciencia posnormal en la sociedad del riesgo. En Acta Sociológica. No. 67, 165-192. <http://dx.doi. org/10.1016/j.acs0.2015.03.001>

»Post, Senja, Nils Bienzeisler y Mareike Lohöfener. 2021. A desire for authoritative science? How citizens' informational needs and epistemic beliefs shaped their views of science, news, and policymaking in the COVID-19 pandemic. En Public Understanding of Science. Vol. 30, no. 5, 496-514. <https://doi.org/10.1177/09636625211005334> 
»Sardar, Ziauddin. 2020. The smog of ignorance: Knowledge and wisdom in postnormal times. En Futures. Vol. 120, e102554. <https://doi.org/https://doi. org/10.1016/j.futures.2020.102554>

" Taddei, Renzo y Cecilia Hidalgo. 2016. Antropología posnormal. En Cuadernos de antropología social. No. 43, 21-32. <https://doi.org/10.34096/cas.i43.2994>

» Uribe-Tirado, Alejandro, Luciano Gallón, Álvaro Monterroza-Ríos, Álvaro Quintero-Posada y Gabriel Vélez-Cuartas. 2020. Datos, información, conocimiento: otra cruel pedagogía del virus. En Uribe-Tirado, Alejandro, Alexander Yarce de los Ríos y Alicia Elena de la Torre Urrea et al. Polifonía para pensar una pandemia. Medellín: Universidad de Antioquia, Fondo Editorial FCSH de la Facultad de Ciencias Sociales y Humanas. p. 67-88.

»Vallor, Shannon. 2018. Technology and the Virtues: A Philosophical Guide to a Future Worth Wanting. New York: Oxford University Press. 
